\title{
Comunicación
}

\section{PERFIL BIOQUÍMICO SANGUÍNEO HEPÁTICO EN COATÍES (Nasua nasua) CRIADOS EN CAUTIVERIO}

\author{
Blood Biochemical Hepatic Profile in the Coati (Nasua nasua) \\ REARED IN CAPTIVITY
}

\author{
Carmen Yupanqui C. ${ }^{1}$, Olga Li E. ${ }^{2,3}$, Walter Silva S. ${ }^{4}$ y Arnaldo Alvarado S. ${ }^{2}$
}

\section{Abstract}

\begin{abstract}
The objective of this study was to establish reference serum values for alanine aminotransferase (ALT), aspartate aminotransferase (AST), bilirubin (total and direct), alkaline phosphatase, total proteins and albumin in captive South American coati (Nasua nasua). A total of 19 coatis (11 males and 8 females) from two zoo parks in the area of Lima, Peru were blood sampled. The coatis were chemically restrained using ketamine clorhidrate (10 mg/kg body weight) and xilacine clorhidrate $(1 \mathrm{mg} / \mathrm{kg}$ body weight) via IM. Blood samples $(4 \mathrm{ml})$ were collected through femoral vein puncture using vacutainer tubes without anticoagulant. The blood chemistry values obtained were ALT: $94.0 \pm 48.5 \mathrm{UI} / \mathrm{L}$, AST: $124.7 \pm 49.4 \mathrm{UI} / \mathrm{L}$, total bilirubin: $0.72 \pm 0.55 \mathrm{mg} / \mathrm{dl}$, direct bilirubin: $0.19 \pm 0.21 \mathrm{mg} / \mathrm{dl}$, indirect bilirubin: $0.52 \pm 0.52 \mathrm{mg} / \mathrm{dl}$, alkaline phosphatase: $46.8 \pm 26.4 \mathrm{UI} / \mathrm{L}$, total proteins: $8.0 \pm 1.1 \mathrm{~g} / \mathrm{dl}$, and albumin: $3.9 \pm 0.5 \mathrm{~g} / \mathrm{dl}$.
\end{abstract}

Key words: serum biochemistry, coati, Nasua nasua

El estudio y conservación de la fauna silvestre está teniendo mayor importancia y se viene creando una conciencia naturalista frente al continuo desarrollo urbano que atenta contra miles de especies animales y su medio ambiente. El coatí (Nasua sp.) es un carnívoro mediano, ampliamente distribuido en el continente americano, donde existen tres especies con sus respectivas áreas geográficas de distribución. Se caracterizan por su peculiar estructura social, formando grupos de hembras y crías, mientras que los machos viven solitarios (Beisiegel, 2001)). El coatí no se encuentra en situación crítica (Braddy, 2003) $\mathrm{y}$ en su medio ambiente cumple un rol de presa y a la vez de predador, pero es importante resaltar que actúa como reservorio silvestre de ciertos parásitos, tales como el Tripanosoma evansi, que afecta al equino (Silva et al., 1996), así como filariosis, que es de caracter zoonótico (Baird y Neafie, 1988).

\footnotetext{
${ }^{1}$ Práctica privada. E-mail: camin19@hotmail.com

${ }^{2}$ Laboratorio de Patología Clínica, FMV-UNMSM.

${ }^{3}$ E-mail: olgalie@hotmail.com

${ }^{4}$ Laboratorio de Anatomía Animal, FMV-UNMSM. E-mail: w_silva01@yahoo.com
} 
En el diagnóstico temprano y certero de las enfermedades que afectan a esta especie, se debe recurrir al examen clínico completo, el examen coprológico y el examen bioquímico sanguíneo y hematológico con el apoyo del laboratorio clínico. El presente estudio se planteó con la finalidad de obtener valores normales de la bioquímica sanguínea hepática en coatíes criados en cautiverio que puedan servir de valores referenciales en la clínica veterinaria de la fauna silvestre.

Se utilizaron coatíes sudamericanos (Nasua nasua) provenientes de dos zoológicos del departamento de Lima, Perú: el zoológico del Patronato del Parque de las Leyendas (PATPAL) $(n=16)$, ubicado en el distrito de San Miguel, y el zoológico del Parque Zonal Sinchi Roca - SERPAR $(\mathrm{n}=3)$, ubicado en el distrito de Comas. La colecta de sangre de los 19 coatíes (11 machos y 8 hembras), mayores de 2 años de edad, se realizó entre noviembre de 2004 y enero de 2005. Los animales tuvieron un peso promedio de $5.2 \mathrm{~kg}$ y se encontraban en condiciones sanitarias aparentemente normales. $\mathrm{La}$ dieta ofrecida era muy similar en ambos zoológicos, y se componía básicamente de una variedad de frutas y verduras, carne y huevo sancochado.

Los animales, días antes del muestreo, fueron trasladados a una zona de acceso restringido, y se sometieron a un ayuno de 12 horas. Se inmovilizaron químicamente con una mezcla de clorhidrato de ketamina $(10 \mathrm{mg} /$ $\mathrm{kg}$ p.v. $)$ y clorhidrato de xilacina $(1 \mathrm{mg} / \mathrm{kg}$ p.v) vía IM. El muestreo se realizó entre las 09:30 y 10:30, colectándose $4 \mathrm{ml}$ de sangre de la vena femoral en tubos tipos vacutainer sin anticoagulante. El suero se obtuvo por centrifugación a 3000 rpm durante 10 minutos. Las muestras se procesaron en el Laboratorio de Patología Clínica de la Facultad de Medicina Veterinaria de la Universidad Nacional Mayor de San Marcos

Se determinaron los niveles séricos de alanina amino transferasa (ALT), aspartato amino transferasa (AST), bilirrubina (total, directa e indirecta), fosfatasa alcalina, proteínas totales y albúmina, empleándose métodos colorimétricos y cinéticos con kits comerciales (Wiener Lab). La lectura de la absorbancia se realizó en un espectrofotómetro UV (Photometro 4010 Manheim Boehringer). Se hizo una estadística descriptiva con los valores obtenidos, estimando la tendencia central (media aritmética) y medidas de dispersión (desviación estándar y rango).

No se disponen de estudios sobre bioquímica sanguínea hepática del coatí sudamericano (Nasua nasua) en el Perú; sin embargo, se disponen de reportes provenientes de exámenes médicos anuales de coatíes aparentemente sanos, pertenecientes de zoológicos de Europa y Estados Unidos de Norteamérica (ISIS, 1999; Denver, 2003).

Los valores de ALT obtenidos (94.0 \pm $48.5 \mathrm{UI} / \mathrm{L}$, Cuadro 1) son menores a los promedios reportados por Denver (2003) (232 \pm 104 UI/L) e ISIS (1999) (218 $\pm 131 \mathrm{UI} / \mathrm{L})$, pero dentro de los amplios rangos observados en dichos estudios. La ALT está presente en el citoplasma de los hepatocitos, y la alteración de la permeabilidad de la membrana hepatocelular resulta en el escape de esta enzima al torrente sanguíneo (Benjamin, 1984; Meyer y Harvey, 1998), de allí que el incremento de ALT es indicador de daño hepatocelular o patología subclínica que afecta la función hepática (Willard et al., 2001), aunque también es producido por acción de algunos fármacos (Benjamin, 1984).

Los valores obtenidos de AST (Cuadro 1) son menores a los reportados por otros autores (ISIS, 1999; Denver, 2003). El aumento de la AST sérica sugiere necrosis hepática o muscular (Sodikoff, 1996), pero también está relacionada a drogas hepatotóxicas (Benjamin, 1984).

Los valores de bilirrubina total y directa (Cuadro 1) guardan similitud con los valores reportados por ISIS (1999) y Denver (2003). 
Cuadro 1. Valores de bioquímica sanguínea para evaluar función hepática de 19 coatíes (Nasua nasua) criados en cautiverio en zoológicos de la ciudad de Lima (20042005)

\begin{tabular}{lcccc}
\hline Variables & Media & $\begin{array}{c}\text { Desviación } \\
\text { estándar }\end{array}$ & $\begin{array}{c}\text { Valor } \\
\text { mínimo }\end{array}$ & $\begin{array}{c}\text { Valor } \\
\text { máximo }\end{array}$ \\
\hline ALT (UI/L) & 94.0 & 48.5 & 27.0 & 218.0 \\
AST (UI/L) & 124.7 & 49.4 & 55.0 & 232.0 \\
Bilirrubina total (mg/dl) & 0.72 & 0.55 & 0.1 & 2.19 \\
Bilirrubina directa (mg/dl) & 0.19 & 0.21 & 0.03 & 0.36 \\
Bilirrubina indirecta (mg/dl) & 0.52 & 0.52 & 0.07 & 2.01 \\
Fosfatasa alcalina (UI/L) & 46.8 & 26.4 & 13.0 & 102.0 \\
Proteínas totales (g/dl) & 8.0 & 1.1 & 5.8 & 10.4 \\
Albúmina (g/dl) & 3.9 & 0.5 & 3.2 & 4.8 \\
\hline
\end{tabular}

La bilirrubina puede acumularse en la sangre o en los tejidos cuando hay incremento en la degradación de los eritrocitos, como sucede en las enfermedades hemolíticas, o en caso de una enfermedad hepática (Slauson y Cooper, 1990).

Los valores obtenidos de fosfatasa alcalina, proteínas totales y albúmina (Cuadro 1) fueron similares a los reportados por ISIS (1999) y Denver (2003). El aumento de fosfatasa alcalina indica obstrucción biliar (Benjamin, 1984) o pancreatitis aguda debido a una colangitis secundaria (Sodikoff, 1996). Por lo general, una hipoproteinemia significativa es el resultado de una hipoalbuminemia (Benjamin, 1984). Esta última se debe a una insuficiencia hepática crónica (Willard et al., 2001), que se presenta en enfermedades hepáticas crónicas como la hepatitis crónica, fibrosis, y neoplasia hepática (Smith, 1990).

Los valores encontrados en el presente estudio han sido comparados con resultados obtenidos en EE.UU. y Europa, de los cuales se desconocen las características de la población, la edad de los animales empleados, así como las condiciones climáticas, de manejo y de alimentación en que se encontraban los animales; efectos que pueden haber influido en los resultados de las pruebas realizadas, y por lo tanto, pueden ser el motivo de la diferencia encontrada en algunos de los parámetros de bioquímica sanguínea hallados en el presente estudio.

\section{Literatura Citada}

1. Baird JK, Neafie RC. 1988. South American brugian filariasis: report of a human infection acquired in Peru. Amer J Trop Med Hyg 39: 185-188.

2. Beisiegel BM. 2001. Notes on the coati, Nasua nasua (Carnivora: Procyonidae) in an Atlantic Forest area. Braz J Biol 61: 689-692.

3. Benjamin M. 1984. Manual de patología clínica en veterinaria. México: Ed. Limusa. 424 p. 
4. Braddy S. 2003. "Nasua nasua". Animal diversity. [Internet], [15 august 2004]. Disponible en: http:// animaldiversity.ummz.umich.edu/site/ accounts/information/Nasua_nasua.html

5. Denver M. 2003. Procyonidae and Viverridae. In: Zoo and wild animal medicine. Fowler ME, Millar RE (eds). 5th ed. Cap. 50. p 516-523. USA: Ed. Elsevier Science.

6. [ISIS] International Species Identification System. 1999. Reference ranges for physiological data values. Clinical pathology record report - ISIS/ In house reference values mammals. [Internet], Available from: http:// www.worldzoo.org

7. Meyer DJ, Harvey JW. 1998. Veterinary laboratory medicine.
Interpretation and diagnosis. $2^{\mathrm{a}}$ ed. Philadelphia, USA: WB Saunders. 368 p.

8. Slauson DO, Cooper BJ. 1990. Mechanisms of disease. A textbook of comparative general pathology. $2^{\mathrm{h}}$ ed. Maryland, USA: Williams \& Wilkins.

9. Smith BP. 1990. Large animal internal medicine. Missouri, USA: Ed. Mosby. $1787 \mathrm{p}$.

10. Sodikoff CH. 1996. Pruebas diagnósticas y de laboratorio en las enfermedades de pequeños animales. $2^{\mathrm{a}} \mathrm{ed}$. Madrid, España: Ed. Mosby. 435 p.

11. Willard MD, Tvedten H, Turnwald GH. 2001. Diagnóstico clínico patológico práctico en los pequeños animales. $3^{\mathrm{a}}$ ed. Buenos Aires, Argentina: Ed. Intermédica. 339 p. 